ESAIM: COCV 20 (2014) 174-189

DOI: $10.1051 / \mathrm{cocv} / 2013060$
ESAIM: Control, Optimisation and Calculus of Variations

www.esaim-cocv.org

\title{
UNIFORM STABILIZATION OF SOME DAMPED SECOND ORDER EVOLUTION EQUATIONS WITH VANISHING SHORT MEMORY
}

\author{
Louis TeBou ${ }^{1}$
}

\begin{abstract}
We consider a damped abstract second order evolution equation with an additional vanishing damping of Kelvin-Voigt type. Unlike the earlier work by Zuazua and Ervedoza, we do not assume the operator defining the main damping to be bounded. First, using a constructive frequency domain method coupled with a decomposition of frequencies and the introduction of a new variable, we show that if the limit system is exponentially stable, then this evolutionary system is uniformly - with respect to the calibration parameter - exponentially stable. Afterwards, we prove uniform polynomial and logarithmic decay estimates of the underlying semigroup provided such decay estimates hold for the limit system. Finally, we discuss some applications of our results; in particular, the case of boundary damping mechanisms is accounted for, which was not possible in the earlier work mentioned above.
\end{abstract}

Mathematics Subject Classification. 93D15, 35L10, 35Q74, 37L15, 74K20.

Received June 1, 2012. Revised December 28, 2012.

Published online December 23, 2013.

\section{INTRODUCTION AND STATEMENTS OF MAIN RESULTS}

Let $H$ be a Hilbert space, and let $A$ be an unbounded coercive operator on $H$ with $A=A^{*}$. Denote $(.,$.$) ,$ the scalar product on $H$, and |.|, the corresponding norm on $H$. Set $V=D\left(A^{\frac{1}{2}}\right)$, and for every $v \in V$, set $\|v\|=\left|A^{\frac{1}{2}} v\right|$. Denote by $V^{\prime}$ the topological dual space of $V$, and let $B: V \rightarrow V^{\prime}$ be a nonnegative operator, viz. $\langle B v, v\rangle \geq 0$ for all $v$ in $V$, where $\langle$,$\rangle denotes the duality product between V$ and $V^{\prime}$. Throughout the paper, it is assumed that $H$ is the pivot space, and that the embeddings $D(A) \subset V \subset H \subset V^{\prime}$ are compact and dense.

For each $\varepsilon>0$, consider the following abstract second order evolution equation

$$
\begin{aligned}
& y_{\varepsilon, t t}+A y_{\varepsilon}+B y_{\varepsilon, t}+\varepsilon A y_{\varepsilon, t}=0, \quad t \in \mathbb{R}, \\
& y_{\varepsilon}(0)=y^{0}, \quad y_{\varepsilon t}(0)=y^{1} .
\end{aligned}
$$

The associated limit system is

$$
\begin{aligned}
& y_{, t t}+A y+B y_{, t}=0, \quad t \in \mathbb{R}, \\
& y(0)=y^{0}, \quad y_{t}(0)=y^{1} .
\end{aligned}
$$

Keywords and phrases. Second order evolution equation, Kelvin-Voigt damping, hyperbolic equations, stabilization, boundary dissipation, localized damping, plate equations, elasticity equations, frequency domain method, resolvent estimates.

1 Department of Mathematics and Statistics, Florida International University, Miami FL 33199, USA. teboul@fiu.edu 
If $y^{0} \in V$, and $y^{1} \in H$, the energy of (1.1) is given for every $t \geq 0$ by

$$
E_{\varepsilon}(t)=\frac{1}{2}\left(\left|y_{\varepsilon, t}(t)\right|^{2}+\left|A^{\frac{1}{2}} y_{\varepsilon}(t)\right|^{2}\right),
$$

and it is a nonincreasing function of the time variable as

$$
E_{\varepsilon}(t)=E_{\varepsilon}(s)-\int_{s}^{t}\left\{\left\langle B y_{\varepsilon, t}(r), y_{\varepsilon t}(r)\right\rangle+\varepsilon\left|A^{\frac{1}{2}} y_{\varepsilon, t}(r)\right|^{2}\right\} \mathrm{d} r, \quad \forall 0 \leq s<t<+\infty .
$$

System (1.1) is motivated by the study of the uniform stabilization of the finite differences or traditional finite element space discretization of the wave equation. Indeed, for such approximation schemes, the energy of the damped wave equation does not decay uniformly with respect to the mesh size; for that to happen, a suitably calibrated vanishing viscosity has to be introduced into the system, e.g. $[15,56,57]$.

The main question that will be dealt with in this paper is the following: Given that the decay of the energy of the limit system (1.2) is exponential, polynomial, or logarithmic, is the exponential/polynomial/logarithmic decay of the energy $E_{\varepsilon}$, as $t \rightarrow \infty$, uniform with respect to $\varepsilon$ ? In other words, given $\varepsilon_{0}>0$, do there exist positive constants $M$ and $\lambda$ such that

$$
E_{\varepsilon}(t) \leq M \mathrm{e}^{-\lambda t} E_{\varepsilon}(0), \quad \forall t \geq 0, \quad \forall 0<\varepsilon \leq \varepsilon_{0}, \quad \forall\left(y^{0}, y^{1}\right) \in V \times H,
$$

or

$$
E_{\varepsilon}(t) \leq \frac{M\left\|\left(y^{0}, y^{1}\right)\right\|_{D\left(\mathcal{A}_{\varepsilon}\right)}^{2}}{(1+t)^{\lambda}}, \quad \forall t \geq 0, \quad \forall 0<\varepsilon \leq \varepsilon_{0}, \quad \forall\left(y^{0}, y^{1}\right) \in D\left(\mathcal{A}_{\varepsilon}\right),
$$

or

$$
E_{\varepsilon}(t) \leq \frac{M\left\|\left(y^{0}, y^{1}\right)\right\|_{D\left(\mathcal{A}_{\varepsilon}\right)}^{2}}{[\log (2+t)]^{2}}, \quad \forall t \geq 0, \quad \forall 0<\varepsilon \leq \varepsilon_{0}, \quad \forall\left(y^{0}, y^{1}\right) \in D\left(\mathcal{A}_{\varepsilon}\right),
$$

where $D\left(\mathcal{A}_{\varepsilon}\right)$ is defined below.

This work was motivated by one of the questions tackled in [14]; indeed the authors of [14] consider, among other things, (1.1) with $B=C^{*} C$, where $C$ is a bounded operator, viz. $C \in \mathcal{L}(H)$. Assuming that the limit system (1.2) is exponentially stable, using an appropriate decomposition of the solution along high and low frequencies, and the fact that $C$ is bounded, they prove (1.5). One may argue that the additional viscoelastic damping makes the stabilization problem much easier, which is true for $\varepsilon$ fixed, but then the decay rate is not uniform with respect to $\varepsilon$, and overdamping may occur as shown in [14]. What makes the study of this stabilization problem interesting is the requirement that the decay rate be uniform with respect to $\varepsilon$ as $\varepsilon$ goes to zero. It is to be noted that in [14], the uniform energy decay estimate (1.5) critically relies on the following two facts:

i) the limit system is exponentially stable;

ii) the damping operator $C$ is bounded.

Consequently if one of those two facts fails, then the method developed in [14], and which is based on Proposition 1 in [19] that establishes an equivalence between observability and stabilization for second order evolution equations with bounded damping operators, becomes inoperative; in particular, the case where the damping operator $B$ is unbounded is left as an open problem therein. It is the intent of the author of the present paper to propose a solution to that open problem; the method that will be developed below to address that problem and which is based on the resolvent estimates will enable us to deal not only with the case where the limit system is exponentially stable, as in [14], but also to deal with situations where the limit system is polynomially or logarithmically stable only; this may happen even for some bounded operators $B$, e.g. [16,31,37, 39, 42, 49,54]. At this stage, it is worth mentioning that the present work as well as [14] are closely related to the earlier works $[56,57]$ where the uniform stabilization of the finite differences space semi-discretization of the wave equation is discussed; in those two papers, the addition of a well-calibrated viscoelastic damping is the key element for the uniform exponential decay of the energy. Indeed, it is shown in $[56,57]$ that without that additional 
damping, the discrete system fails to be uniformly exponentially stable. But as will be seen below, and as it was already observed in [14], the presence of the viscoelastic damping in (1.1) makes the study of the stabilization problem at hand more intricate; in fact, the authors of [14] had to rely on a judicious decomposition of the solution along high and low frequencies in order to prove that the perturbed system is uniformly exponentially stable. In the present work, where the decay estimates will be established through estimates of the resolvent along the imaginary axis, we will decompose the axis into two portions; for the unbounded portion, the extra viscoelastic damping will be enough to get the necessary estimates, while for the bounded portion, we will rely on the introduction of a new variable and the fact that the limit resolvent satisfies the corresponding estimate.

Before stating our main results, we will recast (1.1) as a first-order system. To this end, introduce the Hilbert space on the field $\mathbb{C}$ of complex numbers $\mathcal{H}=V \times H$, equipped with the norm

$$
\|(u, v)\|_{1}^{2} \doteq\|(u, v)\|_{\mathcal{H}}^{2}=\left|A^{\frac{1}{2}} u\right|^{2}+|v|^{2} .
$$

Let $\mathcal{A}_{\varepsilon}$ be the unbounded operator given by

$$
\mathcal{A}_{\varepsilon}=\left(\begin{array}{cc}
0 & I \\
-A-B-\varepsilon A
\end{array}\right)
$$

with:

$$
D\left(\mathcal{A}_{\varepsilon}\right)=\{(u, v) \in V \times V ; A(u+\varepsilon v)+B v \in H\} .
$$

System (1.1) may now be recast as

$$
\begin{aligned}
& Z_{\varepsilon t}=\mathcal{A}_{\varepsilon} Z_{\varepsilon} \\
& Z_{\varepsilon}(0)=\left(\begin{array}{c}
y^{0} \\
y^{1}
\end{array}\right) .
\end{aligned}
$$

We denote by $\mathcal{A}_{0}$ the unbounded limit operator with domain

$$
D\left(\mathcal{A}_{0}\right)=\{(u, v) \in V \times V ; A u+B v \in H\} .
$$

It will be assumed in the sequel that

$$
\exists \lambda_{0}>0:|u| \leq \lambda_{0}\left|A^{\frac{1}{2}} u\right|, \quad \forall u \in V,
$$

and

$$
\exists \mu_{0}>0:\langle B u, u\rangle \leq \mu_{0}^{2}\|u\|^{2}, \quad \forall u \in V .
$$

We can now state our main results:

Theorem 1.1. Let the operators $A$ and $B$ be given as above. Assume that the limit operator $\mathcal{A}_{0}$ generates a $C_{0}$ semigroup of contractions $\left(S_{0}(t)\right)_{t \geq 0}$ on the Hilbert space $\mathcal{H}$ which is exponentially stable. Let $\varepsilon_{0}>0$ be an arbitrary constant. There exist positive constants $M$ and $\lambda$, that eventually depend on $\lambda_{0}, \mu_{0}$, and $\varepsilon_{0}$ only, such that the energy decay estimate (1.5) holds for every solution of (1.1).

Theorem 1.2. Let the operators $A$ and $B$ be given as above. Assume that the limit operator $\mathcal{A}_{0}$ satisfies $i \mathbb{R} \subset \rho\left(\mathcal{A}_{0}\right)$, where $\rho(\mathcal{A})$ denotes the resolvent of $\mathcal{A}$. Suppose that $\mathcal{A}_{0}$ generates a $C_{0}$ semigroup of contractions $\left(S_{0}(t)\right)_{t \geq 0}$ on the Hilbert space $\mathcal{H}$, which is polynomially stable, viz., there are positive constants $M_{0}$ and $\alpha_{0}$ such

$$
\left\|S_{0}(t) Z^{0}\right\|_{\mathcal{H}} \leq \frac{M_{0}\left\|Z^{0}\right\|_{D\left(\mathcal{A}_{0}\right)}}{(1+t)^{\alpha_{0}}}, \quad \forall t \geq 0, \quad Z^{0} \in D\left(\mathcal{A}_{0}\right) .
$$

Set $\varepsilon_{0}=1$. There exist positive constants $M$ and $\lambda$, that eventually depend on $\lambda_{0}$ and $\mu_{0}$ only, such that the energy decay estimate (1.6) holds for every solution of (1.1). 
Theorem 1.3. Let the operators $A$ and $B$ be given as above. Assume that $\mathcal{A}_{0}$ generates a $C_{0}$ semigroup of contractions $\left(S_{0}(t)\right)_{t \geq 0}$ on the Hilbert space $\mathcal{H}$. Suppose that there exists a positive constant $C_{0}$ such that for every $\lambda \in \mathbb{C}$ with $\Re \lambda \in\left[-\frac{\mathrm{e}^{-C_{0}|\Im \lambda|}}{C_{0}}, 0\right]$ and $|\Im \lambda| \geq 1$, one has the resolvent estimate

$$
\left\|\left(\lambda \mathcal{I}-\mathcal{A}_{0}\right)^{-1}\right\|_{\mathcal{L}(\mathcal{H})} \leq C_{0} \mathrm{e}^{C_{0}|\Im \lambda|} .
$$

Set $\varepsilon_{0}=1$. There exists a positive constant $M$, that eventually depends on $\lambda_{0}$ and $\mu_{0}$ only, such that the energy decay estimate (1.7) holds for every solution of (1.1).

Remark 1.4. It is known that when the resolvent estimate (1.14) holds, then the semigroup $\left(S_{0}(t)\right)_{t \geq 0}$ is logarithmically stable $[16,17,31]$; there exists a positive constant $M_{0}$ such that for every positive integer $k$,

$$
\left\|S_{0}(t) Z^{0}\right\|_{\mathcal{H}} \leq \frac{M_{0}\left\|Z^{0}\right\|_{D\left(\mathcal{A}_{0}^{k}\right)}}{[\log (2+t)]^{k}}, \quad \forall t \geq 0, \quad Z^{0} \in D\left(\mathcal{A}_{0}^{k}\right) .
$$

The rest of this paper is organized as follows: in Section 2, we recall some important preliminary results relating the energy decay estimates to resolvent estimates. Section 3 deals with the proofs of Theorems 1.1-1.3, while in Section 4 we discuss several applications of our results and some final remarks.

\section{SOME TECHNICAL LEMMAS}

Lemma $2.1[20,44]$. Let $\mathcal{A}$ be the generator of a bounded $C_{0}$ semigroup $(S(t))_{t \geq 0}$ on a Hilbert space $\mathcal{H}$. Then $(S(t))_{t \geq 0}$ is exponentially stable if and only if:

i) $i \mathbb{R} \subset \rho(\mathcal{A})$, and

ii) $\sup \left\{\left\|(i b-\mathcal{A})^{-1}\right\| ; b \in \mathbb{R}\right\}<\infty$, where $\rho(\mathcal{A})$ denotes the resolvent of $\mathcal{A}$.

Lemma 2.2 [7]. Let $\mathcal{A}$ be the generator of a bounded $C_{0}$ semigroup $(S(t))_{t \geq 0}$ on a Hilbert space $\mathcal{H}$ such that $i \mathbb{R} \subset \rho(\mathcal{A})$, where $\rho(\mathcal{A})$ denotes the resolvent of $\mathcal{A}$. Then $(S(t))_{t \geq 0}$ is polynomially stable, viz., there are positive constants $M$ and $\alpha$ that are independent of the initial data such

$$
\left\|S(t) Z^{0}\right\|_{\mathcal{H}} \leq \frac{M\left\|Z^{0}\right\|_{D(\mathcal{A})}}{(1+t)^{\frac{1}{\alpha}}}, \quad \forall t \geq 0, \quad Z^{0} \in D(\mathcal{A}) .
$$

if and only if

$$
\exists C_{0}>0: \|\left.(i b-\mathcal{A})^{-1}\right|_{\mathcal{L}(\mathcal{H})} \leq C_{0}|b|^{\alpha}, \forall b \in \mathbb{R} \text { with }|b| \geq 1 .
$$

Weaker versions of Lemma 2.2 may be found in [5, 6, 35].

\section{Proofs of Theorems 1.1, 1.2, And 1.3}

As indicated in the introduction, the energy decay estimates will be derived from resolvent estimates. For that derivation, we will rely on Lemma 2.1 for the case of Theorem 1.1 and Lemma 2.2 for the case of Theorem 1.2. First, we shall prove that $\mathcal{A}_{\varepsilon}$ generates a $C_{0}$ semigroup of contractions $\left(S_{\varepsilon}(t)\right)_{t \geq 0}$, then we shall show that $i \mathbb{R} \subset \rho\left(\mathcal{A}_{\varepsilon}\right)$.

We have:

- the operator $\mathcal{A}_{\varepsilon}$ is dissipative as:

$$
\Re\left(\mathcal{A}_{\varepsilon} Z, Z\right)=-\varepsilon\left|A^{\frac{1}{2}} v\right|^{2}-\langle B v, v\rangle \leq 0, \quad \forall Z=(u, v) \in \mathcal{D}\left(\mathcal{A}_{\varepsilon}\right) .
$$

- $\mathcal{I}-\mathcal{A}_{\varepsilon}$ is onto, by Lax-Milgram Lemma, ( $\mathcal{I}$ denotes the identity operator).

Consequently, the operator $\mathcal{A}_{\varepsilon}$ generates a $C_{0}$ semigroup of contractions on $\mathcal{H}$ by Lumer-Phillips theorem [41]; note that $\overline{D\left(\mathcal{A}_{\varepsilon}\right)}=\mathcal{H}$, by [41], Theorem 4.6, page 16 . 
We now observe that the operator $\mathcal{A}_{\varepsilon}$ does not have a compact resolvent even though $\mathcal{A}_{0}$ might have one as we will see in the examples that are discussed later on. This is due to the fact that the extra viscoelastic damping has the same order as the principal operator $A$, thereby precluding the embedding of $D\left(\mathcal{A}_{\varepsilon}\right)$ into $\mathcal{H}$ to be compact. Next, we note that $0 \in \rho\left(\mathcal{A}_{\varepsilon}\right)$. Let $b \in \mathbb{R}$ with $b \neq 0$, the assertion about the resolvent will be established once we prove: i) $\operatorname{Ker}\left(i b-\mathcal{A}_{\varepsilon}\right)=\{\mathbf{0}\}$ and ii) $R\left(i b-\mathcal{A}_{\varepsilon}\right)=\mathcal{H}$, where $\operatorname{Ker}(B)$ stands for the kernel of the operator $B$ and $R(B)$ stands for the range of $B$.

Proof of $i$ ). Let $b$ be a nonzero real number and let $Z=(u, v) \in D\left(\mathcal{A}_{\varepsilon}\right)$ with $\mathcal{A}_{\varepsilon} Z=i b Z$, we shall prove that $Z=\mathbf{0}$. The equation $\mathcal{A}_{\varepsilon} Z=i b Z$ easily yields $\Re\left(\mathcal{A}_{\varepsilon} Z, Z\right)=-\varepsilon\left|A^{\frac{1}{2}} v\right|^{2}-\langle B v, v\rangle=0$; from which one derives $v=0$, thanks to (1.11), and then $u=0$. Hence $Z=\mathbf{0}$.

Proof of ii). For this proof, we borrow some ideas from [36]. Let $b$ be a nonzero real number, and let $U=(f, g) \in$ $\mathcal{H}$. We shall show that there exists $Z=(u, v) \in D\left(\mathcal{A}_{\varepsilon}\right)$ such that $i b Z-\mathcal{A}_{\varepsilon} Z=U$, which may be recast as:

$$
\begin{aligned}
& i b u-v=f \\
& i b v+A(u+\varepsilon v)+B v=g .
\end{aligned}
$$

We may use the first equation in (3.1) to eliminate $v$ in the second one, thereby getting

$$
-b^{2} u+(1+i b \varepsilon) A u+i b B u=g+i b f+\varepsilon A f+B f \in V^{\prime} .
$$

If we set $A_{b \varepsilon}=(1+i b \varepsilon) A+i b B: V \longrightarrow V^{\prime}$, then Lax-Milgram theorem shows that $A_{b \varepsilon}$ is an isomorphism. Further, one checks that $A_{b \varepsilon}^{-1}$ is compact as $A_{b \varepsilon}^{-1}\left(V^{\prime}\right)=V$, and the embedding $V \subset H$ is compact. We may rewrite $(3.2)$ as

$$
u-b^{2} A_{b \varepsilon}^{-1} u=A_{b \varepsilon}^{-1}(g+i b f+\varepsilon A f+B f) .
$$

Thanks to the Fredholm alternative e.g. [8], Theorem VI.6, page 92, solving (3.3) in $H$ amounts to showing that the equation $u-b^{2} A_{b \varepsilon}^{-1} u=0$ has the unique solution $u=0$, or equivalently that $u=0$ is the unique solution of the equation $-b^{2} u+(1+i b \varepsilon) A u+i b B u=0$. Taking the duality product $V^{\prime}-V$ of $u$ and both sides of the latter equation, we get: $-b^{2}|u|^{2}+(1+i b \varepsilon)\left|A^{\frac{1}{2}} u\right|^{2}+i b\langle B u, u\rangle=0$, so that taking the imaginary parts, and keeping in mind that $b \neq 0$, one finds: $\varepsilon\left|A^{\frac{1}{2}} u\right|^{2}+\langle B u, u\rangle=0$; from which one derives $u=0$, thanks to (1.11). Hence ii) holds. Therefore, combining i), ii) and the closed graph theorem, one derives $i \mathbb{R} \subset \rho\left(\mathcal{A}_{\varepsilon}\right)$. One may now invoke the stability theorem in [3] to conclude that the semigroup $\left(S_{\varepsilon}(t)\right)_{t \geq 0}$ is strongly stable. The Proofs of Theorems 1.1-1.3 that follow now will quantify that strong stability according to the stability property satisfied by the limit system.

\subsection{Proof of Theorem 1.1}

According to Lemma 2.1, it remains to show that one has:

$$
\sup \left\{\left\|\left(i b-\mathcal{A}_{\varepsilon}\right)^{-1}\right\|_{\mathcal{L}(\mathcal{H})} ; \quad b \in \mathbb{R}\right\}<\infty, \quad \forall 0<\varepsilon \leq \varepsilon_{0} .
$$

To this end, let $U=(f, g) \in \mathcal{H}$. We shall prove that there exists a constant $C>0$ such that for every $b \in \mathbb{R}$, and every $\varepsilon$ with $0<\varepsilon \leq \varepsilon_{0}$, if $Z=\left(\begin{array}{l}u \\ v\end{array}\right) \in D\left(\mathcal{A}_{\varepsilon}\right)$ satisfies

$$
\left(i b-\mathcal{A}_{\varepsilon}\right) Z=U
$$

then

$$
\|Z\|_{\mathcal{H}} \leq C\|U\|_{\mathcal{H}} .
$$

Normally $Z$ should depend on $\varepsilon$ and $b$, but for simplicity sake, that dependence is omitted. Here and in the sequel, $C$ is a generic constant that may eventually depend on $\lambda_{0}, \mu_{0}$, and $\varepsilon_{0}$ only. 
Denoting by $(.,)_{1}$, the inner product in $\mathcal{H}$, and by $\|.\|_{1}$, the corresponding norm, as introduced in 3.59 , we derive from (3.5):

$$
\left(\left(i b-\mathcal{A}_{\varepsilon}\right) Z, Z\right)_{1}=(U, Z)_{1},
$$

so that taking the real parts, we get

$$
\varepsilon\left|A^{\frac{1}{2}} v\right|^{2}+\langle B v, v\rangle \leq\|U\|_{1}\|Z\|_{1}
$$

Now (3.5) is equivalent to:

$$
\begin{aligned}
& i b u-v=f \\
& i b v+A(u+\varepsilon v)+B v=g .
\end{aligned}
$$

It follows from the first equation in (3.8), and (3.7):

$$
\varepsilon b^{2}\left|A^{\frac{1}{2}} u\right|^{2} \leq 2\left\{\varepsilon\left|A^{\frac{1}{2}} v\right|^{2}+\varepsilon\left|A^{\frac{1}{2}} f\right|^{2}\right\} \leq 2\left(\|U\|_{1}\|Z\|_{1}+\varepsilon_{0}\|U\|_{1}^{2}\right) .
$$

At this stage, we note that if $\underline{\varepsilon b^{2}>1}$, then one derives from (3.9):

$$
\left|A^{\frac{1}{2}} u\right|^{2} \leq 2\left(\|U\|_{1}\|Z\|_{1}+\varepsilon_{0}\|U\|_{1}^{2}\right) .
$$

Taking the inner product in $H$ of $v$ with both sides of the first equation in (3.8), it follows

$$
|v|^{2} \leq|(i b u, v)|+|(f, v)| \leq|(i b u, v)|+C|| U\left\|_{1}\right\| Z \|_{1} .
$$

Taking the duality product $V^{\prime}-V$ of $u$ with both sides of the second equation in (3.8), we derive, thanks to (3.10), (3.7), Cauchy-Schwarz inequality, and (1.12):

$$
\begin{aligned}
|(i b v, u)| & \leq\left|A^{\frac{1}{2}} u\right|^{2}+\varepsilon\left|A^{\frac{1}{2}} u \| A^{\frac{1}{2}} v\right|+|\langle B v, u\rangle|+|(g, u)| \\
& \leq C\left\{\|U\|_{1}\|Z\|_{1}+\|U\|_{1}^{2}+\|U\|_{1}^{\frac{3}{2}}\|Z\|_{1}^{\frac{1}{2}}+\sqrt{\langle B v, v\rangle} \sqrt{\langle B u, u\rangle}\right\} \\
& \leq C\left\{\|U\|_{1}\|Z\|_{1}+\|U\|_{1}^{2}+\|U\|_{1}^{\frac{3}{2}}\|Z\|_{1}^{\frac{1}{2}}+\|U\|_{1}^{\frac{1}{2}}\|Z\|_{1}^{\frac{3}{2}}\right\} .
\end{aligned}
$$

Reporting (3.12) in (3.11), we find

$$
|v|^{2} \leq C\left\{\|U\|_{1}\|Z\|_{1}+\|U\|_{1}^{2}+\|U\|_{1}^{\frac{3}{2}}\|Z\|_{1}^{\frac{1}{2}}+\|U\|_{1}^{\frac{1}{2}}\|Z\|_{1}^{\frac{3}{2}}\right\} .
$$

Combining (3.10) and (3.13), we derive

$$
\|Z\|_{1}^{2} \leq C\left\{\|U\|_{1}\|Z\|_{1}+\|U\|_{1}^{2}+\|U\|_{1}^{\frac{3}{2}}\|Z\|_{1}^{\frac{1}{2}}+\|U\|_{1}^{\frac{1}{2}}\|Z\|_{1}^{\frac{3}{2}}\right\}
$$

so that using Young inequality, (3.6) easily follows from (3.14), provided that $\varepsilon b^{2}>1$. We now turn to the case where $\varepsilon b^{2} \leq 1$. This case is a little bit trickier; first, we will have to make appropriate change of variables, then use the resolvent assumption on the limit system to derive (3.6). To this end, set $w=u+\varepsilon v, \hat{Z}=\left(\begin{array}{c}w \\ v\end{array}\right)$, and $\hat{U}=\left(\begin{array}{c}f+i b \varepsilon v \\ g\end{array}\right)$. We note that $\hat{Z}$ lies in the domain of the limit operator $D\left(\mathcal{A}_{0}\right)$, and $\hat{U} \in \mathcal{H}$; the energy space is the same for the perturbed and limit systems. With those notations, (3.8) becomes

$$
\begin{aligned}
& i b w-v=f+i b \varepsilon v \\
& i b v+A w+B v=g,
\end{aligned}
$$


or equivalently

$$
\left(i b-\mathcal{A}_{0}\right) \hat{Z}=\hat{U}
$$

Thanks to the exponential stability assumption on the limit system, and Lemma 2.1, we know that there exists a positive constant $C$ such that for every real number $b$, one has $\left\|\left(i b-\mathcal{A}_{0}\right)^{-1}\right\|_{\mathcal{L}(\mathcal{H})} \leq C$. Consequently, it follows from (3.16):

$$
\|\hat{Z}\|_{1} \leq C\|\hat{U}\|_{1}
$$

Now, one checks that

$$
\begin{aligned}
\|\hat{U}\|_{1}^{2} & =\left|A^{\frac{1}{2}}(f+i \varepsilon b v)\right|^{2}+|g|^{2} \\
& \leq 2\left|A^{\frac{1}{2}} f\right|^{2}+\varepsilon^{2} b^{2}\left|A^{\frac{1}{2}} v\right|^{2}+|g|^{2} \\
& \leq 2|| U \|_{1}^{2}+2 \varepsilon\left|A^{\frac{1}{2}} v\right|^{2}, \text { since } \varepsilon b^{2} \leq 1 \\
& \leq C\left(\|U\|_{1}^{2}+\|U\|_{1}\|Z\|_{1}\right),
\end{aligned}
$$

and

$$
\begin{aligned}
\|Z\|_{1}^{2} & =\left|A^{\frac{1}{2}} u\right|^{2}+|v|^{2} \\
& \leq 2\left|A^{\frac{1}{2}} w\right|^{2}+2 \varepsilon^{2}\left|A^{\frac{1}{2}} v\right|^{2}+|v|^{2} \\
& \leq 2\|\hat{Z}\|_{1}^{2}+2 \varepsilon_{0}\|U\|_{1}\|Z\|_{1} .
\end{aligned}
$$

The combination of (3.17), (3.18) and (3.19) yields

$$
\|Z\|_{1}^{2} \leq C\left(\|U\|_{1}^{2}+\|U\|_{1}\|Z\|_{1}\right),
$$

from which, one derives (3.6) with the help of Cauchy-Schwarz inequality. This completes the proof of (3.6), and that of Theorem 1.1.

\subsection{Proof of Theorem 1.2}

First, we note that the assumptions on the limit operator $\mathcal{A}_{0}$, and Lemma 2.2 show that there are two positive constants $C_{0}$ and $\alpha=1 / \alpha_{0}$ such that:

$$
\|\left.\left(i b-\mathcal{A}_{0}\right)^{-1}\right|_{\mathcal{L}(\mathcal{H})} \leq C_{0}|b|^{\alpha}, \forall b \in \mathbb{R} \text { with }|b| \geq 1 .
$$

To prove Theorem 1.1, we distinguished two cases: the case $\varepsilon b^{2}>1$, and the case $\varepsilon b^{2} \leq 1$. One might be tempted to use exactly the same two cases in the proof of Theorem 1.2, but then the decay rate would be much weaker than that of the limit system. If one wants to get the same decay rate as in the limit system, then the threshold must involve the exponent $\alpha$ found in (3.21). Let $b \in \mathbb{R}$ with $|b| \geq 1$. Let $U \in \mathcal{H}$ and $Z \in D\left(\mathcal{A}_{\varepsilon}\right)$ satisfy (3.5). We shall prove that there exists a positive constant $C$ such that

$$
\|Z\|_{1} \leq C|b|^{\alpha}|| U \|_{1}, \forall b \in \mathbb{R} \text { with }|b| \geq 1, \quad \forall 0<\varepsilon \leq 1 .
$$

Case $\varepsilon|\boldsymbol{b}|^{2+\alpha}>1$. It follows from (3.9)

$$
\varepsilon|b|^{2+\alpha}\left|A^{\frac{1}{2}} u\right|^{2} \leq 2|b|^{\alpha}\left\{\varepsilon\left|A^{\frac{1}{2}} v\right|^{2}+\varepsilon\left|A^{\frac{1}{2}} f\right|^{2}\right\} \leq 2|b|^{\alpha}\left(\|U\|_{1}\|Z\|_{1}+\|U\|_{1}^{2}\right),
$$

from which one derives

$$
\left|A^{\frac{1}{2}} u\right|^{2} \leq C|b|^{\alpha}\left(\|U\|_{1}\|Z\|_{1}+\|U\|_{1}^{2}\right) .
$$

Proceeding as in the proof of Theorem 1.1, one gets

$$
|v|^{2} \leq C|b|^{\alpha}\left(\|U\|_{1}\|Z\|_{1}+\|U\|_{1}^{2}\right)+C\|U\|_{1}^{\frac{1}{2}}\|Z\|_{1}^{\frac{3}{2}} .
$$


The combination of (3.24) and (3.25) yields

$$
\|Z\|_{1}^{2} \leq C|b|^{\alpha}\left(\|U\|_{1}\|Z\|_{1}+\|U\|_{1}^{2}\right)+C\|U\|_{1}^{\frac{1}{2}}\|Z\|_{1}^{\frac{3}{2}} .
$$

Case $\varepsilon|\boldsymbol{b}|^{2+\alpha} \leq 1$. Let $\hat{U}$ and $\hat{Z}$ be given as in the proof of Theorem 1.1. Then $\hat{U}$ and $\hat{Z}$ satisfy (3.16), so that using (3.21), we find

$$
\|\hat{Z}\|_{1} \leq C_{0}|b|^{\alpha}\|\hat{U}\|_{1}
$$

Thanks to (3.18) and (3.7), one has

$$
\begin{aligned}
\|\hat{U}\|_{1}^{2} & \leq 2\left|A^{\frac{1}{2}} f\right|^{2}+\varepsilon^{2} b^{2}\left|A^{\frac{1}{2}} v\right|^{2}+|g|^{2} \\
& \leq\left. 2\left|\|U\|_{1}^{2}+2 \varepsilon\right| b\right|^{2}\|U\|_{1}\|Z\|_{1} \\
& \leq 2\left(\|U\|_{1}^{2}+|b|^{-\alpha}|| U\left\|_{1}\right\| Z \|_{1}\right), \text { since } \varepsilon|b|^{2+\alpha} \leq 1 .
\end{aligned}
$$

Proceeding as in the proof of Theorem 1.1, and using (3.27) and (3.28), we get the estimate (keeping in mind that $|b| \geq 1)$

$$
\begin{aligned}
\|Z\|_{1}^{2} & \leq 2\|\hat{Z}\|_{1}^{2}+2\|U\|_{1}\|Z\|_{1} \\
& \leq 2 C_{0}^{2} b^{2 \alpha}\|\hat{U}\|_{1}^{2}+2\|U\|_{1}\|Z\|_{1} \\
& \leq 4 C_{0}^{2} b^{2 \alpha}\|U\|_{1}^{2}+\left(4 C_{0}+2\right)|b|^{\alpha}\|U\|_{1}\|Z\|_{1} .
\end{aligned}
$$

Combining (3.26) and (3.29), then applying the Cauchy-Schwarz inequality, one derives (3.22). Using Lemma 2.2, we obtain the claimed energy estimate, which completes the proof of Theorem 1.2.

\subsection{Proof of Theorem 1.3}

Thanks to our resolvent hypothesis, we already know that there exists a positive constant $C_{0}$ such that for every $\lambda \in \mathbb{C}$ with $\Re \lambda \in\left[-\frac{\mathrm{e}^{-C_{0}|\Im \lambda|}}{C_{0}}, 0\right]$ and $|\Im \lambda| \geq 1$, one has the resolvent estimate

$$
\left\|\left(\lambda \mathcal{I}-\mathcal{A}_{0}\right)^{-1}\right\|_{\mathcal{L}(\mathcal{H})} \leq C_{0} \mathrm{e}^{C_{0}|\Im \lambda|} .
$$

We shall find a positive constant $K_{0}$ such that for every $\lambda \in \mathbb{C}$ with $\Re \lambda \in\left[-\frac{\mathrm{e}^{-K_{0}|\Im \lambda|}}{K_{0}}, 0\right]$ and $|\Im \lambda| \geq 1$, one has the resolvent estimate

$$
\left\|\left(\lambda \mathcal{I}-\mathcal{A}_{\varepsilon}\right)^{-1}\right\|_{\mathcal{L}(\mathcal{H})} \leq K_{0} \mathrm{e}^{K_{0}|\Im \lambda|}, \quad \forall 0<\varepsilon \leq 1 .
$$

Once (3.31) is established, the claimed decay estimate follows as in the proof of Theorem 3 in [9]. So it remains to prove (3.31). To this end, let $U=(f, g) \in \mathcal{H}$, and let $L_{0}>C_{0}$, with $C_{0}$ as in (3.30). Let $\lambda \in \mathbb{C}$ with

$$
\Re \lambda \in\left[-\frac{\mathrm{e}^{-L_{0}|\Im \lambda|}}{L_{0}}, 0\right] \text { and }|\Im \lambda| \geq 1 .
$$

As in the proof of Theorem 1.1 above, introduce $Z=(u, v) \in D\left(\mathcal{A}_{\varepsilon}\right)$ such that

$$
\lambda Z-\mathcal{A}_{\varepsilon} Z=U
$$

which is equivalent to

$$
\begin{aligned}
& \lambda u-v=f \\
& \lambda v+A(u+\varepsilon v)+B v=g .
\end{aligned}
$$

The inner product of $Z$ with both sides of (3.33) yields

$$
\lambda\|Z\|_{1}^{2}-\left(\mathcal{A}_{\varepsilon} Z, Z\right)_{1}=(U, Z)_{1},
$$


so that taking the real parts, we derive

$$
\varepsilon\left|A^{\frac{1}{2}} v\right|^{2}+\langle B v, v\rangle \leq\|U\|_{1}\|Z\|_{1}+|\Re \lambda|\|Z\|_{1}^{2} .
$$

Applying the operator $A^{\frac{1}{2}}$ to both sides of the first equation in (3.34), and using (3.35), we obtain

$$
\varepsilon|\lambda|^{2}\left|A^{\frac{1}{2}} u\right|^{2} \leq 2\left\{\varepsilon\left|A^{\frac{1}{2}} v\right|^{2}+\varepsilon\left|A^{\frac{1}{2}} f\right|^{2}\right\} \leq 2\left(\|U\|_{1}\|Z\|_{1}+|\Re \lambda|\|Z\|_{1}^{2}+\|U\|_{1}^{2}\right) .
$$

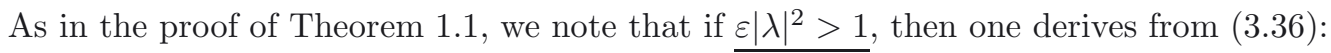

$$
\left|A^{\frac{1}{2}} u\right|^{2} \leq 2\left(\|U\|_{1}\|Z\|_{1}+|\Re \lambda|\|Z\|_{1}^{2}+\|U\|_{1}^{2}\right) .
$$

Taking the inner product in $H$ of $v$ with both sides of the first equation in (3.34), it follows

$$
|v|^{2} \leq|(\lambda u, v)|+|(f, v)| \leq|(\lambda u, v)|+C|| U\left\|_{1}|| Z\right\|_{1} .
$$

Taking the duality product $V^{\prime}-V$ of $u$ with both sides of the second equation in (3.34), we derive, thanks to (3.37), (3.35), Cauchy-Schwarz inequality, and (1.12):

$$
\begin{aligned}
|(\lambda v, u)| & \leq\left|A^{\frac{1}{2}} u\right|^{2}+\varepsilon\left|A^{\frac{1}{2}} u\right|\left|A^{\frac{1}{2}} v\right|+|\langle B v, u\rangle|+|(g, u)| \\
& \leq 2\left|A^{\frac{1}{2}} u\right|^{2}+\varepsilon\left|A^{\frac{1}{2}} v\right|^{2}+\sqrt{\langle B v, v\rangle} \sqrt{\langle B u, u\rangle}+C|| U\left\|_{1}\right\| Z \|_{1} \\
& \leq C\left\{\|U\|_{1}\|Z\|_{1}+\|U\|_{1}^{2}\right\}+\|U\|_{1}^{\frac{1}{2}}\|Z\|_{1}^{\frac{3}{2}}+\left(3|\Re \lambda|+|\Re \lambda|^{\frac{1}{2}}\right)\|Z\|_{1}^{2} .
\end{aligned}
$$

Reporting (3.39) in (3.38), we find

$$
|v|^{2} \leq C\left\{\|U\|_{1}\|Z\|_{1}+\|U\|_{1}^{2}\right\}+\|U\|_{1}^{\frac{1}{2}}\|Z\|_{1}^{\frac{3}{2}}+\left(3|\Re \lambda|+|\Re \lambda|^{\frac{1}{2}}\right)\|Z\|_{1}^{2} .
$$

Combining (3.37) and (3.40), we derive

$$
\|Z\|_{1}^{2} \leq C\left\{\|U\|_{1}\|Z\|_{1}+\|U\|_{1}^{2}\right\}+\|U\|_{1}^{\frac{1}{2}}\|Z\|_{1}^{\frac{3}{2}}+\left(5|\Re \lambda|+|\Re \lambda|^{\frac{1}{2}}\right)\|Z\|_{1}^{2} .
$$

Choosing $L_{0}$ large enough in (3.32), it follows that $5|\Re \lambda|+|\Re \lambda|^{\frac{1}{2}} \leq 1 / 4$; combining that with Young inequality, one finds

$$
\|Z\|_{1}^{2} \leq C\|U\|_{1}^{2}
$$

We got (3.42) by assuming $\varepsilon|\lambda|^{2}>1$. We now investigate the case $\varepsilon|\lambda|^{2} \leq 1$. Let $w=u+\varepsilon v$, and $\check{f}=f+\varepsilon \lambda v$, and set $\check{Z}=(w, v)$ and $\check{U}=(\check{f}, g)$. One easily checks that $\check{Z} \in D\left(\mathcal{A}_{0}\right)$, and $\check{U} \in \mathcal{H}$ satisfy the equation

$$
\left(\lambda-\mathcal{A}_{0}\right) \check{Z}=\check{U},
$$

so that using (3.30), one gets

$$
\|\check{Z}\|_{1} \leq C_{0} \mathrm{e}^{C_{0}|\Im \lambda|}\|\check{U}\|_{1} .
$$

Now on the one hand, one has the estimate

$$
\begin{aligned}
\|\check{U}\|_{1}^{2} & \leq 2\left|A^{\frac{1}{2}} f\right|^{2}+\varepsilon^{2}|\lambda|^{2}\left|A^{\frac{1}{2}} v\right|^{2}+|g|^{2} \\
& \leq 2|| U\left\|_{1}^{2}+2 \varepsilon|\lambda|^{2}\right\| U\left\|_{1}|| Z\right\|_{1}+2 \varepsilon|\lambda|^{2}|\Re \lambda|\|Z\|_{1}^{2} \\
& \leq 2\left(\|U\|_{1}^{2}+\|U\|_{1}\|Z\|_{1}+|\Re \lambda|\|Z\|_{1}^{2}\right), \text { since } \varepsilon|\lambda|^{2} \leq 1 .
\end{aligned}
$$

On the other hand, one has, thanks to 3.59 , the estimate

$$
\begin{aligned}
\|Z\|_{1}^{2} & \leq 2\|\check{Z}\|_{1}^{2}+2 \varepsilon^{2}\left|A^{\frac{1}{2}} v\right|^{2} \\
& \leq 2 C_{0}^{2} \mathrm{e}^{2 C_{0}|\Im \lambda|}\|\check{U}\|_{1}^{2}+2\|U\|_{1}\|Z\|_{1}+2|\Re \lambda|\|Z\|_{1}^{2} .
\end{aligned}
$$


Accounting for (3.60) in (3.61), we find

$$
\begin{aligned}
\|Z\|_{1}^{2} \leq & 4 C_{0}^{2} \mathrm{e}^{2 C_{0}|\Im \lambda|}\left(\|U\|_{1}^{2}+\|U\|_{1}\|Z\|_{1}+|\Re \lambda|\|Z\|_{1}^{2}\right) \\
& +2\|U\|_{1}\|Z\|_{1}+2|\Re \lambda|\|Z\|_{1}^{2} .
\end{aligned}
$$

Thanks to Young inequality, one derives the estimates

$$
4 C_{0}^{2} \mathrm{e}^{2 C_{0}|\Im \lambda|}\|U\|_{1}\|Z\|_{1} \leq \frac{1}{4}\|Z\|_{1}^{2}+16 C_{0}^{4} \mathrm{e}^{4 C_{0}|\Im \lambda|}\|U\|_{1}^{2} .
$$

and

$$
2\|U\|_{1}\|Z\|_{1} \leq \mathrm{e}^{2 L_{0}|\Im \lambda|}\|U\|_{1}^{2}+\mathrm{e}^{-2 L_{0}|\Im \lambda|}\|Z\|_{1}^{2} .
$$

We also note that for large enough $L_{0}$, one has the estimates

$$
4 \mathrm{e}^{2 C_{0}|\Im \lambda|}|\Re \lambda| \leq \frac{1}{16}, \quad \mathrm{e}^{-2 L_{0}|\Im \lambda|} \leq \frac{1}{16}, \text { and } 2|\Re \lambda| \leq \frac{1}{16} .
$$

Reporting (3.63)-(3.65) in (3.62), we obtain, with some algebra

$$
\|Z\|_{1}^{2} \leq \frac{16}{9}\left(20 C_{0}^{4} \mathrm{e}^{4 C_{0}|\Im \lambda|}+\mathrm{e}^{2 L_{0}|\Im \lambda|}\right)\|U\|_{1}^{2} .
$$

At this stage, we note that $C_{0}$ is a large constant (cf. e.g. $\left.[9,31]\right)$; this explains replacing $C_{0}^{2} \mathrm{e}^{2 C_{0}|\Im \lambda|}$ with $C_{0}^{4} \mathrm{e}^{4 C_{0}|\Im \lambda|}$ to get $20 C_{0}^{4} \mathrm{e}^{4 C_{0}|\Im \lambda|}$. Therefore, choosing $L_{0} \geq 8 \sqrt{10} C_{0}^{2} / 3$, it holds

$$
\|Z\|_{1}^{2} \leq L_{0}^{2} \mathrm{e}^{2 L_{0}|\Im \lambda|}\|U\|_{1}^{2} .
$$

Hence, it suffices to choose $K_{0}=L_{0}$.

\section{Applications}

In this section we shall discuss some examples of application of our theorems. Throughout this section, $\Omega$ denotes a bounded domain in $\mathbb{R}^{N}$ with smooth enough boundary, subscripts following a comma stand for differentiation, and we use the Einstein summation convention on repeated indices. Further, $\partial_{i}$ stands for $\partial / \partial x_{i}$, $|u|_{r}$ denotes $\|u\|_{L^{r}(\Omega)}$ for $1 \leq r \leq+\infty$. We assume that the boundary $\Gamma$ of $\Omega$ satisfies: $\Gamma=\Gamma_{c} \cup \Gamma_{u}$, with $\bar{\Gamma}_{c} \cap \bar{\Gamma}_{u}=\emptyset$, where $\Gamma_{c}$ stands for the controlled portion of $\Gamma$ and meas $\left(\Gamma_{c}\right)>0$, while $\Gamma_{u}$ corresponds to the uncontrolled portion, and meas $\left(\Gamma_{u}\right)>0$, for simplification purposes.

\subsection{The wave equation with boundary damping}

Consider the damped wave equation:

$$
\left\{\begin{array}{l}
u, t t-\partial_{i}\left(b_{i j}(x) \partial_{j} u\right)=0 \text { in } \Omega \times(0, \infty) \\
b_{i j}(x) \partial_{j} u \nu_{i}+u_{t}=0 \text { on } \Sigma_{c}, \quad u=0 \text { on } \Sigma_{u}=\partial \Omega \times(0, T) \\
u(0)=u^{0} ; \quad u_{, t}(0)=u^{1} \text { in } \Omega
\end{array}\right.
$$

where here and in the sequel, $\nu$ denotes the unit vector pointing into the exterior of $\Omega$, the coefficients $\left(b_{i j}\right)_{i, j}$, satisfy:

$$
b_{i j} \in C^{1}(\bar{\Omega}) ; \quad b_{i j}=b_{j i}, \quad \forall i, j=1,2, \ldots, N,
$$

and

$$
\exists a_{0}>0: b_{i j}(x) z_{i} z_{j} \geq a_{0} z_{i} z_{i}, \quad \forall(x, z) \in \bar{\Omega} \times \mathbb{R}^{N} .
$$


If one sets $V=\left\{v \in H^{1}(\Omega) ; v=0\right.$ on $\left.\Gamma_{u}\right\}$, then one can show that for $\left(u^{0}, u^{1}\right) \in V \times L^{2}(\Omega)$, one has $u \in C([0, \infty) ; V) \cap C^{1}\left([0, \infty) ; L^{2}(\Omega)\right)$.

For every $t \in[0, T]$, set

$$
E(t)=\frac{1}{2} \int_{\Omega}\left\{\left|u_{t}(x, t)\right|^{2}+\left(b_{i j}(x) \partial_{j} u(x, t) \partial_{i} u(x, t)\right\} \mathrm{d} x .\right.
$$

The energy $E$ is a nonincreasing function of the time variable, as we have the dissipation law:

$$
E(s)=E(t)+\int_{s}^{t} \int_{\Gamma_{c}}\left|u_{, t}(\gamma, t)\right|^{2} \mathrm{~d} \gamma \mathrm{d} r, \quad \forall 0 \leq s<t<\infty .
$$

It is now well known that if the boundary of $\Omega$ is $C^{\infty}$, the coefficients $b_{i j} \in C^{\infty}(\Omega)$, and $\Gamma_{c}$ satisfies the geometric control condition of Bardos-Lebeau-Rauch [4]: there exists a time $T>0$ such that every ray of geometric optics meets $\Gamma_{c} \times(0, T)$, then the energy $E$ satisfies the exponential decay estimate:

$$
E(t) \leq M \mathrm{e}^{-\lambda t} E(0), \quad \forall t \geq 0 .
$$

Many other authors proved the exponential decay estimates under various conditions on the boundary of $\Omega$, the coefficients $b_{i j}$ and the feedback control support $\Gamma_{c}$, e.g. $[10,13,21,22,24,25,27,29,30,37,38,45-48,59-61]$.

Now, consider the perturbed problem, with all parameters as above:

$$
\left\{\begin{array}{l}
u_{\varepsilon, t t}-\partial_{i}\left(b_{i j}(x) \partial_{j} u_{\varepsilon}\right)-\varepsilon \partial_{i}\left(b_{i j}(x) \partial_{j} u_{\varepsilon, t}\right)=0 \text { in } \Omega \times(0, \infty) \\
b_{i j}(x) \partial_{j}\left(u_{\varepsilon}+\varepsilon u_{\varepsilon, t}\right) \nu_{i}+u_{\varepsilon, t}=0 \text { on } \Sigma_{c}=\Gamma_{c} \times(0, \infty), \\
u_{\varepsilon}=0 \text { on } \Sigma_{u}=\Gamma_{u} \times(0, \infty) \\
u_{\varepsilon}(0)=u^{0} ; \quad u_{\varepsilon, t}(0)=u^{1} \text { in } \Omega .
\end{array}\right.
$$

We are going to apply Theorem 1.1 to System (4.6) to prove that its energy given by

$$
E_{\varepsilon}(t)=\frac{1}{2} \int_{\Omega}\left\{\left|u_{\varepsilon, t}(x, t)\right|^{2}+\left(b_{i j}(x) \partial_{j} u_{\varepsilon}(x, t) \partial_{i} u_{\varepsilon}(x, t)\right\} \mathrm{d} x,\right.
$$

and which satisfies, for all $0 \leq s<t<\infty$, the dissipation law

$$
E_{\varepsilon}(s)=E_{\varepsilon}(t)+\int_{s}^{t} \int_{\Gamma_{c}}\left|u_{\varepsilon, t}(\gamma, t)\right|^{2} \mathrm{~d} \gamma \mathrm{d} r+\varepsilon \int_{s}^{t} \int_{\Omega} b_{i j}(x) \partial_{j} u_{\varepsilon, t}(x, t) \partial_{i} u_{\varepsilon t}(x, t) \mathrm{d} x \mathrm{~d} s,
$$

decays exponentially, uniformly with respect to the perturbation parameter $\varepsilon$. To this end, set $H=L^{2}(\Omega)$, $V=\left\{v \in H^{1}(\Omega) ; v=0\right.$ on $\left.\Gamma_{u}\right\}, A u=-\partial_{i}\left(b_{i j} \partial_{j} u\right)$ with $D(A)=\{u \in V ; A u \in H\},\langle B u, v\rangle=\int_{\Gamma_{c}} u \bar{v} \mathrm{~d} \gamma$, for all $u, v \in V$. Then according to the hypotheses on the coefficients, the operator $A$ is coercive with $A^{*}=A$. The operator $B$ is nonnegative, well-defined on $V$ with values in the dual space $V^{\prime}$ thanks to Riesz representation theorem. If we also set $y_{\varepsilon}=u_{\varepsilon}$, then (4.6) may be recast as the abstract equation (1.1). Further, it can be shown that the operators $A$ and $B$ satisfy (1.11) and (1.12) respectively. Therefore, if $\Gamma_{c}$ satisfies the BardosLebeau-Rauch geometric control condition (GCC), then the semigroup generated by the limit operator $\mathcal{A}_{0}$ is exponentially stable thanks to (4.5) and Lemma 2.1; applying Theorem 1.1, one derives that the perturbed energy $E_{\varepsilon}$ decays exponentially, uniformly with respect to the perturbation parameter $\varepsilon$, as the time variable $t$ goes to infinity. On a different note, it can be shown that $\mathcal{A}_{0}$ has a compact resolvent, e.g. [22], Lemmas 7.7, 7.8 , but the perturbed operator $\mathcal{A}_{\varepsilon}$ does not.

Now, if $\Gamma_{c}$ does not satisfy (GCC), then the exponential decay (4.5) for the energy $E$ fails. In this case, it is known that for smoother initial data, and under certain conditions on $\Gamma_{c}$, polynomial decay estimates for the energy $E$ hold when $A u=-\Delta u$, [43], while logarithmic decay estimates for the energy $E$ hold for any $\Gamma_{c}$ with a nonzero measure, e.g. $[16,32]$. In the former case, applying Theorem 1.2, one derives uniform polynomial decay estimates for the energy $E_{\varepsilon}$, and in the latter case, the application of Theorem 1.3 provides a uniform logarithmic decay for the energy $E_{\varepsilon}$. 


\subsection{The elasticity equations with boundary damping}

Consider the damped elasticity system

$$
\left\{\begin{array}{l}
y_{i, t t}-\sigma_{i j, j}=0 \text { in } \Omega \times(0, \infty) \\
\sigma_{i j} \nu_{j}+y_{i, t}=0 \text { on } \Gamma_{c} \times(0, \infty) \quad y_{i}=0 \text { on } \Gamma_{u} \times(0, \infty) \\
y_{i}(0)=y_{i}^{0}, \quad y_{i, t}(0)=y_{i}^{1}, \quad i=1,2, \ldots, N
\end{array}\right.
$$

where the elasticity stress tensor $\left(\sigma_{i j}\right)$ is given by

$$
\sigma_{i j}=\sigma_{i j}(y)=a_{i j k l} \varepsilon_{k l}
$$

with $\left(\varepsilon_{k l}\right)$ defined by

$$
\varepsilon_{k l}=\varepsilon_{k l}(y)=\frac{1}{2}\left(y_{k, l}+y_{l, k}\right)
$$

is the strain tensor. The $a_{i j k l}$ are the elasticity coefficients. They satisfy the symmetry properties

$$
a_{i j k l}=a_{j i l k}=a_{k l i j}, \quad \forall i, j, k, l .
$$

Throughout the paper we assume that the $a_{i j k l}$ depend on the space variable $x$ but not on time, and that they are continuously differentiable, and satisfy the ellipticity condition

$$
\exists a_{0}>0: a_{i j k l} u_{i j} u_{k l} \geq a_{0} u_{i j} u_{k l}
$$

for all second order symmetric tensors $\left(u_{i j}\right)$.

Under the above assumptions on the coefficients, and for all $i,\left(y_{i}^{0}, y_{i}^{1}\right) \in V \times L^{2}(\Omega)$, it is well-known that System (4.8) has a unique weak solution $y \in \mathcal{C}\left([0, \infty) ; V^{N}\right) \cap \mathcal{C}^{1}\left([0, \infty) ;\left[L^{2}(\Omega)\right]^{N}\right)$.

Introduce the energy

$$
E(t)=\frac{1}{2} \int_{\Omega}\left\{\left|y_{, t}(x, t)\right|^{2}+\left(\sigma_{i j} \varepsilon_{i j}\right)(x, t)\right\} \mathrm{d} x, \quad \forall t \geq 0 .
$$

The energy $E$ is a nonincreasing function of the time variable $t$ and its derivative satisfies

$$
E^{\prime}(t)=-\int_{\Gamma_{c}}\left|y_{, t}(\gamma, t)\right|^{2} \mathrm{~d} \gamma, \quad \forall t \geq 0
$$

It is well-known that if $\Gamma_{c}$ is an appropriate portion of the boundary, and some structural constraints are imposed on the coefficients $a_{i j k l}$, then the energy $E$ satisfies an exponential decay estimate of type (4.5), e.g. $[2,23,26,37,40,48]$. We shall now show that the perturbed system

$$
\left\{\begin{array}{l}
y_{\varepsilon i, t t}-\sigma_{i j, j}\left(y_{\varepsilon}\right)-\varepsilon \sigma_{i j, j}\left(y_{\varepsilon, t}\right)=0 \text { in } \Omega \times(0, \infty) \\
\sigma_{i j}\left(y_{\varepsilon}+\varepsilon y_{\varepsilon, t}\right) \nu_{j}+y_{\varepsilon i, t}=0 \text { on } \Gamma_{c} \times(0, \infty) \quad y_{\varepsilon i}=0 \text { on } \Gamma_{u} \times(0, \infty) \\
y_{\varepsilon i}(0)=y_{i}^{0}, \quad y_{\varepsilon i, t}(0)=y_{i}^{1}, \quad i=1,2, \ldots, N
\end{array}\right.
$$

where the elasticity stress tensor $\left(\sigma_{i j}\right)$ is now given by

$$
\sigma_{i j}\left(y_{\varepsilon}\right)=a_{i j k l} \varepsilon_{k l}\left(y_{\varepsilon}\right),
$$

is uniformly exponentially stable. To this end, set $H=\left[L^{2}(\Omega)\right]^{N}$ and $V=\left\{u \in\left[H^{1}(\Omega)\right]^{N} ; u=0\right.$ on $\left.\Gamma_{u}\right\}$. If we set $A u=-\sigma_{i j, j}(u)$, with $D(A)=\{u \in V ; A u \in H\}$. Define the operator $B$ by $\langle B u, v\rangle=\int_{\Gamma_{c}} u \cdot \bar{v} d \Gamma$, then $B$ is nonnegative, well-defined according to Riesz representation theorem, and one can check that $A$ and $B$ satisfy (1.11) and (1.12) respectively. Moreover (4.12) may be recast as (1.1). On the other hand, knowing that for an appropriate portion of the boundary, the limit system is exponentially stable, it follows from Theorem 1.1 that the perturbed system is uniformly, with respect to the perturbation parameter, exponentially stable. 


\subsection{The Euler-Bernoulli equation with unbounded locally distributed damping}

Let $a \in L^{\infty}(\Omega)$, be a nonnegative function satisfying:

$$
\exists a_{0}>0: a(x) \geq a_{0}, \quad \text { a.e. } x \in \omega,
$$

where $\omega$ is an appropriate open set contained in $\Omega$.

Consider the following damped Euler-Bernoulli equation

$$
\left\{\begin{array}{l}
w_{, t t}+\Delta^{2} w-\operatorname{div}\left(a \nabla w_{, t}\right)=0 \text { in } \Omega \times(0, \infty) \\
w=\frac{\partial w}{\partial \nu}=0 \text { on } \Gamma \times(0, \infty) \\
w(0)=y^{0} \text { in } \Omega \\
w_{, t}(0)=y^{1} \text { in } \Omega .
\end{array}\right.
$$

System (4.14) corresponds to the clamped plate equation with structural damping when $a \equiv 1$, and $N=2$, [12]. Condition (4.13) ensures that the damping term $-\operatorname{div}\left(a \nabla w_{, t}\right)$ is effective on the set $\omega$.

Let $\left\{y^{0}, y^{1}\right\} \in H_{0}^{2}(\Omega) \times L^{2}(\Omega)$. System (4.14) is then well-posed in the space $H_{0}^{2}(\Omega) \times L^{2}(\Omega)$.

Introduce the energy

$$
E(t) \equiv E(w ; t)=\frac{1}{2} \int_{\Omega}\left\{|w, t(x, t)|^{2}+|\Delta w(x, t)|^{2}\right\} \mathrm{d} x, \quad \forall t \geq 0 .
$$

The energy $E$ is a nonincreasing function of the time variable $t$ and we have for almost every $t \geq 0$,

$$
E^{\prime}(t)=-\int_{\Omega} a(x)\left|\nabla w_{, t}(x, t)\right|^{2} \mathrm{~d} x
$$

The decay estimates of the energy of plate equations with a locally distributed frictional damping of the form $a y_{t}$ or $a g\left(y_{t}\right)$, for an appropriate nonlinear function $g$, are well-known, e.g. [1,11,18, 19, 34, 50, 52, 58, 62]. Concerning the system (4.14) with a locally distributed structural damping, it was recently shown in [55] that, if $\omega$ satisfies the geometric constraint described in [22,33] or [34], then its energy, given by (4.15), satisfies an exponential decay estimate of type (4.5).

Introduce the perturbed system

$$
\left\{\begin{array}{l}
w_{\varepsilon, t t}+\Delta^{2} w_{\varepsilon}-\operatorname{div}\left(a \nabla w_{\varepsilon, t}\right)+\varepsilon \Delta^{2} w_{\varepsilon, t}=0 \text { in } \Omega \times(0, \infty) \\
w_{\varepsilon}=\frac{\partial w_{\varepsilon}}{\partial \nu}=0 \text { on } \Gamma \times(0, \infty) \\
w_{\varepsilon}(0)=y^{0} \text { in } \Omega \\
w_{\varepsilon, t}(0)=y^{1} \text { in } \Omega .
\end{array}\right.
$$

If we set $H=L^{2}(\Omega), V=H_{0}^{2}(\Omega), A=\Delta^{2}$ with clamped boundary conditions, $\langle B u, v\rangle=\int_{\Omega} a(x) \nabla u \cdot \nabla \bar{v} \mathrm{~d} x$, for all $u, v \in V$. Then, the operator $A$ is coercive with $A^{*}=A$, and the operator $B$ is nonnegative, well-defined on $V$ with values in the dual space $V^{\prime}$. If we also set $y_{\varepsilon}=w_{\varepsilon}$, then (4.17) may be recast as the abstract equation (1.1). Further, it can be shown that the operators $A$ and $B$ satisfy (1.11) and (1.12) respectively. On the other hand, we also know that the unbounded operator $\mathcal{A}_{0}$ associated with (4.14) generates an exponentially stable semigroup [55]. The application of Theorem 1.1 shows that the perturbed energy $E_{\varepsilon}=E\left(w_{\varepsilon} ;\right.$.) decays exponentially, uniformly with respect to $\varepsilon$. 


\subsection{Final remarks}

1) Although the perturbation $\varepsilon A y_{\varepsilon, t}$ is more relevant physically, given its viscoelastic character, one could have, from a purely mathematical viewpoint, used a more general operator $\hat{A}$ having properties similar to those of the operator $A$. But the operator $\hat{A}$ should further be required that $V=D\left(A^{\frac{1}{2}}\right)$ be densely embedded in $D\left(\hat{A}^{\frac{1}{2}}\right)$, so that $A^{-1} \hat{A} u$ lies in $V$ for all $u$ in $D\left(\hat{A}^{\frac{1}{2}}\right)$; in particular that requirement is fulfilled by $\hat{A}=A^{\alpha}$ for any $\alpha \leq 1$. If one were to use the perturbation $\varepsilon A^{\alpha}$ instead, the interesting values of $\alpha$ would be those in the interval $(0,1]$. Indeed, for $\alpha \leq 0$ the perturbation is either compact $(\alpha<0)$, or it can be easily absorbed by the energy.

2) It was noted in the introduction that overdamping may occur, meaning that the exponential decay does not hold uniformly with respect to $\varepsilon$ as $\varepsilon$ goes to infinity. This fact was demonstrated in the case of a bounded damping operator $B$ in [14]. It can also be established in the case of an unbounded damping operator $B$. For instance, if we choose $B=A$, which corresponds to the Kelvin-Voigt damping, then the operator $\mathcal{A}_{0}$ is known to generate an analytic semigroup; so, it can be shown that the semigroup generated by the operator $\mathcal{A}_{\varepsilon}$ is also analytic; the proof of this fact just follows the same algorithm devised above in the proof of the exponential decay of the semigroup. Now, we are going to show that a branch of the eigenvalues of the operator $\mathcal{A} \varepsilon$ behaves so badly as $\varepsilon \rightarrow \infty$ that exponential decay of the energy fails to be uniform as $\varepsilon \rightarrow \infty$. To this end, remember that the operator $A$ is elliptic, self-adjoint, and has a compact resolvent; therefore the spectrum of the operator $A$ is discrete and we assume that it is given by the increasing sequence $\left\{\mu_{j}^{2} ; \mu_{j}>0, \quad j \geq 1\right\}$. The $j^{\text {th }}$ eigenvalue $\lambda_{j \varepsilon}$ of the operator $\mathcal{A}_{\varepsilon}$ (keep in mind that we have chosen $B=A$ here) satisfies the quadratic equation: $\lambda^{2}+(1+\varepsilon) \mu_{j}^{2} \lambda+\mu_{j}^{2}=0$. Consequently, one has:

$$
\lambda_{j \varepsilon}^{ \pm}=\frac{(1+\varepsilon) \mu_{j}^{2}}{2}\left(-1 \pm \sqrt{1-\frac{4}{(1+\varepsilon)^{2} \mu_{j}^{2}}}\right),
$$

so that

$$
\lambda_{j \varepsilon}^{+} \sim-\frac{1}{1+\varepsilon}, \quad \text { as }(1+\varepsilon) \mu_{j} \rightarrow \infty
$$

Therefore this branch of the spectrum of $\mathcal{A}_{\varepsilon}$ approaches zero as $\varepsilon \rightarrow \infty$, thereby precluding the uniform exponential decay of the energy.

Acknowledgements. The author thanks the anonymous referees for their valuable comments, and suggestions that helped to improve the presentation of this paper.

\section{REFERENCES}

[1] F. Alabau-Boussouira, Piecewise multiplier method and nonlinear integral inequalities for Petrowsky equation with nonlinear dissipation. J. Evol. Equ. 6 (2006) 95-112.

[2] F. Alabau and V. Komornik, Boundary observability, controllability, and stabilization of linear elastodynamic systems. SIAM J. Control Optim. 37 (1999) 521-542.

[3] W. Arendt, C. J. K. Batty, Tauberian theorems and stability of one-parameter semigroups. Trans. Amer. Math. Soc. 306 (1988) 837-852.

[4] C. Bardos, G. Lebeau and J. Rauch, Sharp sufficient conditions for the observation, control and stabilization from the boundary. SIAM J. Control Optim. 30 (1992) 1024-1065.

[5] A. Bátkai, K.-J. Engel, J. Prüss and R. Schnaubelt, Polynomial stability of operator semigroups. Math. Nachr. 279 (2006) $1425-1440$.

[6] C.J.K. Batty and T. Duyckaerts, Non-uniform stability for bounded semi-groups on Banach spaces. J. Evol. Equ. 8 (2008) $765-780$.

[7] A. Borichev and Y. Tomilov, Optimal polynomial decay of functions and operator semigroups. Math. Annal. 347 (2010) $455-478$.

[8] H. Brezis, Analyse fonctionnelle. Théorie et Applications. Masson, Paris (1983).

[9] N. Burq, Décroissance de l'énergie locale de l'équation des ondes pour le problème extérieur et absence de résonance au voisinage du réel. Acta Math. 180 (1998) 1-29. 
[10] G. Chen, Control and stabilization for the wave equation in a bounded domain. SIAM J. Control Optim. 17 (1979) 66-81.

[11] G. Chen, S.A. Fulling, F.J. Narcowich and S. Sun, Exponential decay of energy of evolution equations with locally distributed damping. SIAM J. Appl. Math. 51 (1991) 266-301.

[12] G. Chen and D.L. Russell, A mathematical model for linear elastic systems with structural damping. Quart. Appl. Math. 39 (1981/1982) 433-454.

[13] F. Conrad and B. Rao, Decay of solutions of the wave equation in a star-shaped domain with nonlinear boundary feedback. Asymptotic Anal. 7 (1993) 159-177.

[14] S. Ervedoza, E. Zuazua, Uniform exponential decay for viscous damped systems. Advances in phase space analysis of partial differential equations. Progr. Nonlinear Differential Equ. Appl. vol. 78. Birkhäuser Boston, Inc., Boston, MA (2009) 95-112.

[15] S. Ervedoza and E. Zuazua, Uniformly exponentially stable approximations for a class of damped systems. J. Math. Pures Appl. 91 (2009) 20-48.

[16] X. Fu, Longtime behavior of the hyperbolic equations with an arbitrary internal damping. Z. Angew. Math. Phys. 62 (2011) 667-680.

[17] X. Fu, Logarithmic decay of hyperbolic equations with arbitrary small boundary damping. Commun. Partial Differ. Equ. 34 (2009) 957-975.

[18] R.B. Guzmán and M. Tucsnak, Energy decay estimates for the damped plate equation with a local degenerated dissipation. Systems Control Lett. 48 (2003) 191-197.

[19] A. Haraux, Une remarque sur la stabilisation de certains systèmes du deuxième ordre en temps. Portugal. Math. 46 (1989) $245-258$.

[20] F.L. Huang, Characteristic conditions for exponential stability of linear dynamical systems in Hilbert spaces. Annal. Differ. Equ. 1 (1985) 43-56.

[21] V. Komornik, Rapid boundary stabilization of the wave equation. SIAM J. Control Optim. 29 (1991) $197-208$.

[22] V. Komornik, Exact controllability and stabilization. The multiplier method, RAM. Masson and John Wiley, Paris (1994).

[23] V. Komornik and V. Boundary stabilization of isotropic elasticity systems. Control of partial differential equations and applications (Laredo, 1994), vol. 174. Lect. Notes Pure and Appl. Math. Dekker, New York (1996) 135-146.

[24] V. Komornik, Rapid boundary stabilization of linear distributed systems. SIAM J. Control Optim. 35 (1997) 1591-1613.

[25] V. Komornik and E. Zuazua, A direct method for the boundary stabilization of the wave equation. J. Math. Pures Appl. 69 (1990) 33-54.

[26] J. Lagnese, Boundary stabilization of linear elastodynamic systems. SIAM J. Control Opt. 21 (1983) $968-984$.

[27] J. Lagnese, Decay of solutions of wave equations in a bounded region with boundary dissipation. J. Differ. Equ. 50 (1983) $163-182$

[28] J. Lagnese, Boundary Stabilization of Thin Plates, vol. 10. SIAM Stud. Appl. Math. Philadelphia, PA (1989).

[29] I. Lasiecka and D. Tataru, Uniform boundary stabilization of semilinear wave equations with nonlinear boundary damping. Differ. Integral Equ. 6 (1993) 507-533.

[30] I. Lasiecka and R. Triggiani, Uniform stabilization of the wave equation with Dirichlet or Neumann feedback control without geometrical conditions. Appl. Math. Optim. 25 (1992) 189-224.

[31] G. Lebeau, Equation des ondes amorties. Algebraic and geometric methods in mathematical physics (Kaciveli, 1993). Math. Phys. Stud. Kluwer Acad. Publ., Dordrecht 19 (1996) 73-109.

[32] G. Lebeau and L. Robbiano, Stabilisation de l'équation des ondes par le bord. Duke Math. J. 86 (1997) 465-491.

[33] J.-L. Lions, Contrôlabilité exacte, Perturbations et Stabilisation des Systèmes Distribués, vol. 8 of RMA. Masson, Paris (1988).

[34] K. Liu, Locally distributed control and damping for the conservative systems. SIAM J. Control and Opt. 35 (1997) $1574-1590$.

[35] Z. Liu and B. Rao, Characterization of polynomial decay rate for the solution of linear evolution equation. Z. Angew. Math. Phys. 56 (2005), 630-644.

[36] K. Liu and B. Rao, Exponential stability for the wave equations with local Kelvin-Voigt damping. Z. Angew. Math. Phys. 57 (2006) 419-432.

[37] P. Martinez, Ph.D. Thesis, University of Strasbourg (1998).

[38] P. Martinez, Boundary stabilization of the wave equation in almost star-shaped domains. SIAM J. Control Optim. 37 (1999) $673-694$.

[39] M. Nakao, Decay of solutions of the wave equation with a local degenerate dissipation. Israel J. Math. 95 (1996) $25-42$.

[40] A. Osses, A rotated multiplier applied to the controllability of waves, elasticity, and tangential Stokes control. SIAM J. Control Optim. 40 (2001) 777-800.

[41] A. Pazy, Semigroups of Linear Operators and Applications to Partial Differential Equations, vol. 44 of Appl. Math. Sci. Springer-Verlag, New York (1983).

[42] K. D. Phung, Polynomial decay rate for the dissipative wave equation. J. Differ. Equ. 240 (2007) 92-124.

[43] K. D. Phung, Boundary stabilization for the wave equation in a bounded cylindrical domain. Discrete Contin. Dyn. Syst. 20 (2008) 1057-1093.

[44] J. Prüss, On the spectrum of $C_{0}$-semigroups. Trans. Amer. Math. Soc. 284 (1984) 847-857.

[45] J.P. Quinn, D.L. Russell, Asymptotic stability and energy decay rates for solutions of hyperbolic equations with boundary damping. Proc. Roy. Soc. Edinburgh Sect. A 77 (1977) 97-127.

[46] D.L. Russell, Controllability and stabilizability theory for linear partial differential equations: recent progress and open questions. SIAM Rev. 20 (1978) 639-739. 
[47] L.R. Tcheugoué Tébou, Sur la stabilisation de l'équation des ondes en dimension 2. C. R. Acad. Sci. Paris Ser. I Math. 319 (1994) 585-588.

[48] L.R. Tcheugoué Tébou, On the stabilization of the wave and linear elasticity equations in 2-D. Panamer. Math. J. 6 (1996) $41-55$.

[49] L.R. Tcheugoué Tébou, On the decay estimates for the wave equation with a local degenerate or nondegenerate dissipation. Portugal. Math. 55 (1998) 293-306.

[50] L.R. Tcheugoué Tébou, Stabilization of the wave equation with localized nonlinear damping J.D.E. 145 (1998) 502-524.

[51] L.R. Tcheugoué Tébou, Well-posedness and energy decay estimates for the damped wave equation with $\mathrm{L}^{r}$ localizing coefficient, Commun. in P.D.E. 23 (1998) 1839-1855.

[52] L.R. Tcheugoué Tébou, Energy decay estimates for the damped Euler-Bernoulli equation with an unbounded localizing coefficient. Portugal. Math. 61 (2004) 375-391.

[53] L.R. Tcheugoue Tebou, On the stabilization of dynamic elasticity equations with unbounded locally distributed dissipation. Differ. Integral Equ. 19 (2006) 785-798.

[54] L. Tebou, Stabilization of the elastodynamic equations with a degenerate locally distributed dissipation. Syst. Control Lett. 56 (2007) 538-545.

[55] L. Tebou, Well-posedness and stabilization of an Euler-Bernoulli equation with a localized nonlinear dissipation involving the p-Laplacian. DCDS A 32 (2012) 2315-2337.

[56] L.R. Tcheugoué Tébou and E. Zuazua, Uniform exponential long time decay for the space finite differences semi-discretization of a locally damped wave equation via an artificial numerical viscosity. Numerische Mathematik 95 (2003) 563-598.

[57] L.T. Tebou and E. Zuazua, Uniform boundary stabilization of the finite differences space discretization of the $1-d$ wave equation. Adv. Comput. Math. 26 (2007) 337-365.

[58] M. Tucsnak, Semi-internal stabilization for a non-linear Bernoulli-Euler equation. Math. Methods Appl. Sci. 19 (1996) 897-907.

[59] A. Wyler, Stability of wave equations with dissipative boundary conditions in a bounded domain. Differential Integral Equ. 7 (1994) 345-366.

[60] E. Zuazua, Robustesse du feedback de stabilisation par contrôle frontière. C. R. Acad. Sci. Paris Ser. I Math. 307 (1988) $587-591$.

[61] E. Zuazua, Uniform stabilization of the wave equation by nonlinear boundary feedback. SIAM J. Control Optim. 28 (1990) $466-477$.

[62] E. Zuazua, Exponential decay for the semilinear wave equation with locally distributed damping. Commun. P.D.E. 15 (1990) 205-235.

[63] E. Zuazua, Exponential decay for the semilinear wave equation with localized damping in unbounded domains. J. Math. Pures. Appl. 70 (1991) 513-529. 